\title{
Associated costs of mitigation-driven translocation in small lizards
}

\author{
Rafael Barrientos ${ }^{1, * * *, * * *}$, Rodrigo Megía-Palma ${ }^{2,3, * * *, * * * *}$
}

\begin{abstract}
Mitigation-driven translocations represent an increasingly common management solution to reduce animal mortality and habitat loss caused by human development. Although they currently outnumber other translocation types, there is a lack of scientific approaches to evaluate the outcome of this management tool. We designed an experimental translocation with two groups of translocated males and two of control males of a small (6-14 g) lizard (totaling 120 individuals). Our results suggest that translocated individuals covered longer distances (53 vs. $18 \mathrm{~m}$ ) from their respective release points in one month (on average), although this distance diminished over time. Displacing longer distances was associated with a body condition impoverishment and an increase in parasitization by ectoparasites. To the best of our knowledge, this is the first study that finds a positive relationship between covering longer distances and an increase in the number of mites. This was also explained by the initial mite load that lizards had, suggesting that controlling the infestation by mites is energetically demanding for lizards, being traded by locomotor activity. At least for those individuals in poorer body condition, we recommend the implementation of soft release (gradually accustoming individuals to their new environment by previously releasing them into controlled conditions) and deparasitization before accomplishing a mitigationdriven translocation.
\end{abstract}

Keywords: corrective measures, environmental impact, habitat loss, homing behaviour, human development, Psammodromus algirus, road ecology, translocation.

\section{Introduction}

Human activities like overexploitation, agricultural development, urban expansion or infrastructure building currently threaten the survival of three quarters of the 82000 species assessed by the International Union for Conservation of Nature (Maxwell et al., 2016). The human footprint will continue growing in the coming decades, as global infrastructure network is expected to continue its expansion in the coming years (Laurance et al., 2014; Meijer et al.,

1 - Road Ecology Lab, Department of Biodiversity, Ecology and Evolution, Faculty of Biology, Complutense University of Madrid, Madrid, Spain

2 - Universidad de Alcalá (UAH), School of Pharmacy, Department of Biomedicine and Biotechnology, Parasitology Area, A.P. 20 Campus Universitario, Alcalá de Henares, E-28805, Madrid, Spain

3 - Functional Biodiversity (FBIO), CIBIO - Centro de Investigação em Biodiversidade e Recursos Genéticos, Universidade do Porto, Vairão, Portugal

${ }^{*}$ Corresponding author; e-mail: rbarrientos@ucm.es

**ORCID: https://orcid.org/0000-0002-1677-3214

*** Both authors contributed equally to this work

**** ORCID: https://orcid.org/0000-0003-1038-0468
2018). Infrastructures can impact wildlife in many ways, like increasing accessibility to natural areas, triggering urban development, and causing direct mortality or barrier effects (Forman et al., 2003; van der Ree, Smith and Grilo, 2015).

Mitigation-driven translocations (i.e., moving animals away from the path of development projects; also called short-distance translocations) attempt to reduce animal mortality and to compensate habitat loss caused by human activities as individuals are moved to new, undisturbed areas (Germano et al., 2015). They can involve species of any conservation status (Germano et al., 2015). These projects have traditionally responded to management needs, and currently outnumber and receive more funding than purely conservation-driven translocations (Germano et al., 2015). However, the success of mitigation-driven translocations is rarely monitored (but see Reinert and Rupert, 1999; Sullivan, Kwiatkowski and Chutt, 2004; Brown, Bishop and Brooks, 2009), which implies that their benefits for conservation remain unclear to date (Germano et al., 
2015). Experimental approaches allow practitioners to evaluate different aspects of translocations in a scientifically rigorous manner (Tetzlaff, Sperry and DeGregorio, 2019), but only a small set of carefully-designed studies have allowed progress in evidence-based conservation (Germano and Bishop, 2009; Germano et al., 2015; Taylor et al., 2017; Tetzlaff et al., 2019). More studies focused on gaining the knowledge needed to improve the success of future translocations are needed to achieve true evidence-based conservation (Tuberville et al., 2005; Taylor et al., 2017). These studies should incorporate experimental designs including the use of controls, a priori hypothesis testing or exploring advantages and disadvantages of potential alternatives (e.g., Tuberville et al., 2005; Taylor et al., 2017). One of the main difficulties that practitioners face before carrying out a translocation, which is common to all taxa, is precisely the lack of baseline information on the parameters that determine establishment success (Berger-Tal, Blumstein and Swaisgoo, 2020).

We assessed the impact of translocation on males of a common Mediterranean lizard compared to controls. We expected that translocation drives males to search for a new territory in their novel habitat, while control individuals remain in the same place where they were captured (and released). Consequently, we expect that (i) translocated individuals move farther distances from the release point than nontranslocated ones (Reinert and Rupert, 1999; Sullivan et al., 2004; Tuberville et al., 2005; Brown et al., 2009). This active search for an empty territory will entail costs: (ii) a short-term impoverishment of body condition (Matthews, 2003); and, (iii) an increase in parasitization intensity by mites either by encountering more infested lizards during their search, or by increasing their exposure to the mites that are present on the ground and vegetation, and/or by reduction of available energy resources to fight parasite infestations because of increased movement (Wu et al., 2019; Wieczorek et al., 2020).

\section{Material and methods}

Study species and study area

Psammodromus algirus (Linnaeus, 1758) is a medium-sized (snout-vent length, SVL, 60-80 mm; 6-14 g), short-lived (5 years) lizard, widespread in shrub and woodland Mediterranean habitats from the Iberian Peninsula (Salvador, 2015). It is considered an active forager with a territorial behavior that tends to increase with age in males (Belliure et al., 1996; Martín and López, 1999). Our study was located at 'El Pardo' (Spain; 40³1' N, 0347' W; $650 \mathrm{~m}$ elevation), a typical shrub-woodland Mediterranean area, where our study species reaches high densities (up to 178 individuals/ha, Salvador, 2015). During the breeding season of 2018 we sampled lizards in two separate areas $>200 \mathrm{~m}$ from each other, which is above the homing distance of medium-sized lacertids (see Strijbosch, van Rooy and Voesenek, 1983). We focused on males to remove the likely confounding effects of sex in the use of the space, which is known to differ between males and females in lacertids (e.g., Lewis and Saliva, 1987; Wieczorek et al., 2020). In total, we sampled 120 adult males, 60 per area, that were divided in 4 groups of 30 males. Two of these groups, one per area, were translocated and reciprocally released into the opposite area (i.e., 'treatment'), and the remaining two groups (also one per area), were released in their original location (i.e., 'control'). One of these areas was adjacent $(0-150 \mathrm{~m})$ to a road (9,050 vehicles per day). We captured (including recaptures) lizards during 18 sampling days, between 24th of April and 27th of June. We extended the recapture effort in 5 more sampling days until 6th of July.

\section{Sampling protocol}

Lizards were collected by using a rod with a noose that tightens around the neck of the lizards, a technique widely used to catch lizards unharmed (e.g., Álvarez-Ruiz et al., 2018). Males were carried to the lab in individual cotton bags to collect additional data under controlled conditions. Lizards were weighed with a digital balance to the nearest centigram. The SVL of the lizards was measured with a ruler to the nearest millimetre as a value of body size. The lizards were toe-clipped and assigned an individual code. Being fully aware that this is an invasive tagging, it is the one that, in small lizards, produces less stress in the medium and long term as evidenced by the methodological work of Langkilde and Shine (2006). These authors concluded that the levels of stress produced by toe-clipping in lizards would not be different from those suffered by lizards in the wild due to predation attempts (Langkilde and Shine, 2006). Because $P$. algirus is a non-arboreal species, this marking does not imply a reduction in its habitual behaviour, and it agrees with the ethical premises of animal experimentation (Perry et al., 2011), when necessary to carry out scientific studies (Buchanan et al., 2012). 


\section{Distance moved}

We measured the 'distance moved' as the Euclidean distance between the point where the individual was released and the point where it was recaptured by using geographic information system. Recaptures in the first 14 days after the release were not included in our analyses.

\section{Body condition}

We calculated a 'body condition' index as the residuals of the regression between $\log _{10}$-transformed values of both body mass and body size (e.g., Dunlap and Mathies, 1993). We included the length of the tail in the regression because $61 \%$ of the lizards in our sample had regenerated tails and this fact can bias the relation between body size and mass. Positive values of the body condition index correspond to lizards being fatter than the sample mean, and negative values are thinner lizards than average.

\section{Quantification of mite parasitization}

We counted 'mites' (ectoparasites) in the field immediately after the lizards were captured (and recaptured) with a magnifying $(\times 10)$ glass (Álvarez-Ruiz et al., 2018). We searched for parasites at the base of the tail because it is the place where they concentrate the most (Álvarez-Ruiz et al., 2018).

\section{Statistical analyses}

We compared the differences in elapsed days between capture and recapture between control and translocated individuals with a two-way ANOVA where the interaction between treatment and area was also considered. Distance moved by the lizards was right skewed (most were small to medium values) and best fitted a Gaussian model after its $\log _{10}$-transformation. The rest of response variables did fit well to Gaussian models without any transformation. The parametric assumptions of normality, homoscedasticity, skewness, and kurtosis were checked on the residual errors of all the models. We carried out three analyses: (i) 'Distance moved' (between the point where the individual was released and the point where it was recaptured). We included as continuous explanatory predictors 'body size', 'body condition' and intensity of parasitization by 'mites' at capture, 'date' of capture, and 'elapsed days' between capture and recapture. As categorical predictors we included the treatment (control vs. translocated), the area and their interaction; (ii) 'Change in body condition' (the value at recapture minus the value at capture, corrected by tail size). We included as continuous explanatory predictors 'body size', 'initial body condition' and intensity of parasitization by 'mites' at capture, 'date' of capture, 'elapsed days' between capture and recapture, and 'distance moved' ( $\log _{10}$ transformed). We included the same categorical predictors and their interaction as above; (iii) 'Change in parasitization by mites' (the value at recapture minus the value at capture). We included as continuous explanatory predictors 'body size', 'body condition' and intensity of parasitization by 'mites' at capture, 'date' of capture, 'elapsed days' between capture and recapture, and 'distance moved'. We included the same categorical predictors and their interaction as above. We checked the multicollinearity of all the models by means of the variance inflation factor (VIF), and also confirmed the normality and the homoscedasticity of the model residuals.

We applied a multimodel inference approach using the R-package 'MuMIn' (Barton, 2013). For this, we considered sufficiently informative all the models with $\triangle \mathrm{AICc}$ $\leqslant 4$ (Burnham and Anderson, 2004). We used model averaging to obtain a final model and calculate the relative importance of each predictor. Only the models that included the effect (i.e., conditional average) were considered to calculate the significance $(\alpha<0.05)$ of the predictors and their z-standardized $\beta$ coefficient \pm standard error. The resulting final models were cross-validated using a k-fold split of 3 in the R-package 'DAAG' (Maindonald, Braun and Braun, 2015). Finally, we calculated the percentage of the variance explained by each significant predictor by means of their sum of squares. Mean values \pm their standard errors are presented hereafter.

\section{Results}

\section{Distance moved}

We recaptured 40 lizards (20 controls, 20 translocated). The elapsed days between capture and recapture were $29.30 \pm 1.38$, not differing between treatment groups $\left(\mathrm{F}_{1,36}=0.02, P=\right.$ $0.87)$ or areas $\left(\mathrm{F}_{1,36}=0.26, P=0.61\right)$, thus confirming that sampling effort and likelihood of recapture were similar between sampling areas. Lizards moved between 3 and 325 meters $($ mean $\pm \mathrm{SE}=35.41 \pm 8.46 \mathrm{~m})$. The multimodel inference approach produced 16 likely models for the distance moved (supplementary table S1). The most important predictors were the treatment (importance $=0.96, \beta=-0.15 \pm$ $0.05 ; \mathrm{z}=2.70, P=0.007)$, and the number of elapsed days between capture and recapture (importance $=0.89, \beta=0.02 \pm 6^{*} 10^{-3} ; \mathrm{z}=$ 2.39, $P=0.017$ ). Thus, translocated lizards moved longer distances shortly after release (fig. 1a). Our results suggested a similar effect of the experiment between areas (importance $=$ $0.09, \beta=-0.043 \pm 0.054 ; \mathrm{z}=0.76, P=0.44$ ) Downloaded from Brill. com04/26/2023 11:22:04AM 
(supplementary fig. S1). The cross-validation of the final model reaffirmed the significant predictors that had been suggested by the multimodel inference approach (table 1).
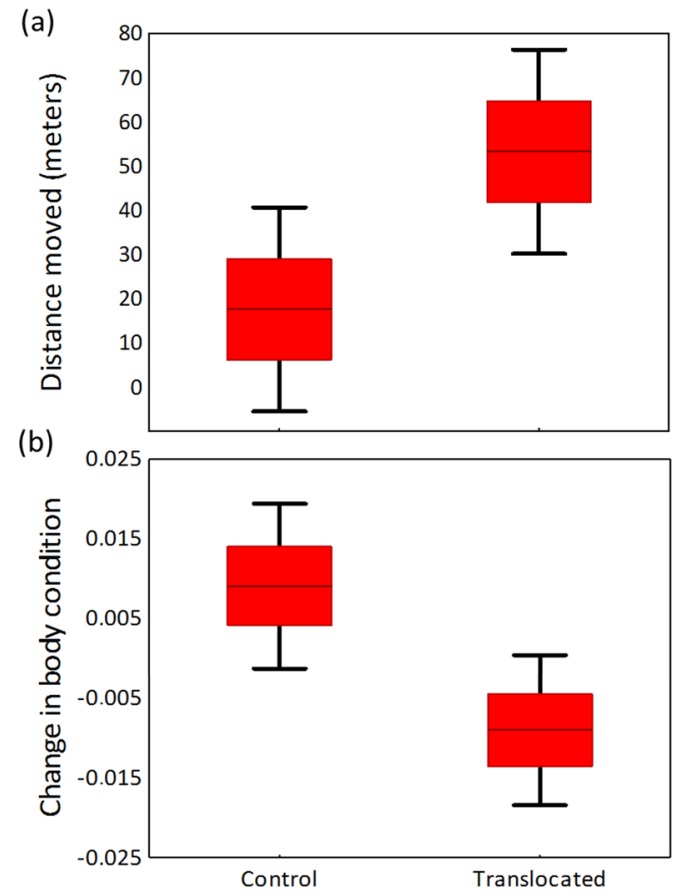

Figure 1. Plots according to model averaging and crossvalidation (see table 1) showing the significant effect of the translocation experiment on (a) the distance moved $\left(\mathrm{F}_{1,37}\right.$ $=8.53, P=0.006)$ and (b) the change in body condition $\left(\mathrm{F}_{1,36}=4.16, P=0.048\right)$ of the males of Psammodromus algirus. Box shows mean $\pm \mathrm{SE}$ and whisker confidence intervals $(95 \%)$.

\section{Change in body condition}

Lizards tended to lose weight during the breeding season as the mean value of change in body condition (residual value) was negative $(-0.00008 \pm 0.004)$. In terms of body mass (grams), lizards lose in average $0.09 \pm 0.10$ grams (mean $0.92 \%$ of the initial weight). The multimodel inference approach produced 13 likely models (supplementary table S2) and suggested that the translocation had a significant effect on the change in body condition of the lizards (importance $=0.77, \beta=7.25^{*} 10^{-3} \pm$ $\left.3.39 * 10^{-3} ; \mathrm{z}=2.07, P=0.038\right)$. Thus, control lizards improved their body condition $(0.009 \pm$ 0.005), whereas translocated ones impoverished it $(-0.009 \pm 0.004)$ (fig. 1b). Indeed, the maximum weight lost by a lizard was 1.82 grams (19.7\% of its initial weight) in an individual translocated close to the road. As well as in the previous variable, the effect of the treatment was independent of the area (importance $=0.03$, $B=-1.87^{*} 10^{-3} \pm 3.15^{*} 10^{-3} ; \mathrm{z}=0.57, P=$ 0.57 ) (supplementary fig. S1). Important predictors for the change in body condition were the number of elapsed days between capture and recapture (importance $=0.96, \beta=1.12 * 10^{-3} \pm$ $\left.4.08 * 10^{-4} ; \mathrm{z}=2.67, P=0.008\right)$, and the distance covered by the individuals (importance $=$ $0.69, \beta=-1.37 * 10^{-4} \pm 6.87 * 10^{-5} ; \mathrm{z}=1.94$, $P=0.053)$. The farther a lizard travelled,

Table 1. Cross-validation ( $\mathrm{k}$-fold $=3$ ) of the final model obtained by multimodel inference for $\log _{10}$-distance moved, change in mite intensity, and change in body condition. Significant predictors are shown in bold $(\alpha=0.05)$. Sum of squares (SS) are also shown.

\begin{tabular}{|c|c|c|c|c|c|}
\hline Distance moved & df & F-value & $P$-value & SS & $\%$ variance \\
\hline Treatment & 1 & 8.53 & 0.006 & 0.96 & 16.5 \\
\hline Elapsed days & 1 & 5.94 & 0.019 & 0.67 & 11.5 \\
\hline Residuals & 37 & & & 4.18 & 71.9 \\
\hline \multicolumn{6}{|c|}{ Change in body condition } \\
\hline Treatment & 1 & 4.16 & 0.048 & 0.001 & 7.4 \\
\hline Elapsed days & 1 & 8.05 & 0.007 & 0.003 & 14.2 \\
\hline Distance moved & 1 & 8.23 & 0.007 & 0.003 & 14.6 \\
\hline Residuals & 36 & & & 0.128 & 63.8 \\
\hline \multicolumn{6}{|c|}{ Change in parasitization by mites } \\
\hline Distance moved & 1 & 7.54 & 0.009 & 1695 & 9.8 \\
\hline Mites_initial & 1 & 32.38 & $<0.001$ & 7281 & 42.1 \\
\hline Residuals & 37 & & & 8318 & 48.1 \\
\hline
\end{tabular}


(a)

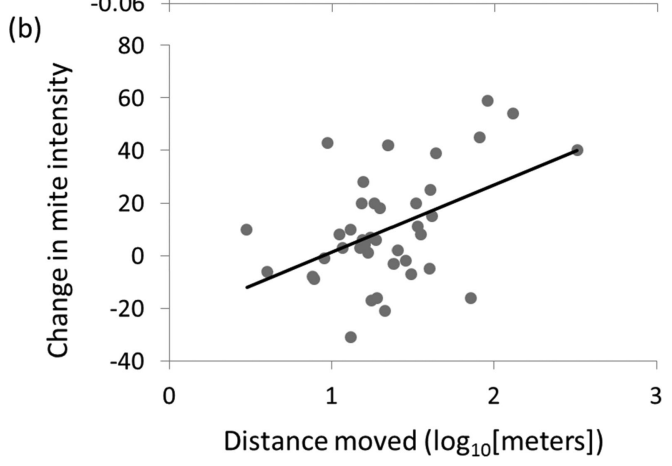

Figure 2. Regression plots showing the relationship between the distance moved by the lizards and the change in (a) body condition, and (b) intensity of mite infestation between capture and recapture.

the worse its body condition became (fig. 2a), but the body condition improved over time. The cross-validation of a model including these three variables confirmed their significance as predictors for the change in body condition (table 1).

\section{Change in parasitization by mites}

The prevalence of haematophagus mites in males of $P$. algirus from El Pardo was 95\% $(114 / 120)$ at capture. The initial number of mites was $19.94 \pm 1.34$ per infested lizard. The change in mite load ranged from -31 to 59 between captures. In general lizards tended to increase their mite load since the mean change was positive $(10.12 \pm 3.33)$. The model averaging approach produced 12 likely models (supplementary table S3). The initial intensity of mites was the best predictor for the change in mite intensity (importance $=1.00, \beta=-1.10 \pm$ $0.24 ; \mathrm{z}=4.37, P=0.001)$, followed by the distance travelled (importance $=0.95, \beta=18.9 \pm$ $7.01 ; \mathrm{z}=2.60, P=0.009)$. Cross-validation of a final model that included these two predictors showed that lizards increased their mite intensity when they moved longer distances (Figure $2 \mathrm{~b}$ ), but this increase was lower if they had a higher number of mites at the beginning of the treatment.

\section{Discussion}

We found increased movements in translocated individuals, likely related to exploratory movements in the search for a new territory. Our results are consistent with telemetry-based studies of mitigation-driven translocations (e.g., Reinert and Rupert, 1999; Sullivan et al., 2004; Tuberville et al., 2005; Brown et al., 2009). This higher motor activity may imply costs for translocated lizards, since they reduced their body condition compared to controls. Furthermore, we demonstrated for the first time that mitigation-driven translocations increase the intensity of parasitization by ectoparasites (mites, in our case). The idea that the ectoparasite load might increase as a cost associated with increased mobility has been frequently suggested (e.g., Salvador et al., 1996; Wu et al., 2019), and only recently demonstrated with ticks (Wieczorek et al., 2020). However, whether ectoparasite load increases in the lizards as a function of encounter rate between lizards and ectoparasites provoked by the travels of the host within their home range, or if contrarily, ectoparasites can replicate within the host as a direct trade-off between immune and motor functions remains obscure. The first seems likely for the case of hard ticks (i.e., fam. Ixodidae), which usually are heteroxenous parasites that require infesting different host taxa to complete their reproductive cycle (Eisen et al., 2004). However, haematophagous mites that infest $P$. algirus in El Pardo (MegíaPalma, unpubl. data), are homoxenous, completing their whole reproductive cycle on the lizards (Reichenow, 1920). Thus, the second scenario of a mite load increase as a function of energetic trade-offs in the host is more likely. 
Translocated animals may face a more stressful environment than controls (reviewed in Teixeira et al., 2007). In vertebrates, the activation of the hypothalamic-pituitary axis in response to stress increases both the mobilization of fat reserves and the activity of the individual as a survival response (Cote et al., 2006). Nevertheless, if food is not accessed because translocated individuals still do not know the resource distribution in their new habitat or the individual is facing a long-term stress, the allocation of body fats to motor activity may be traded by the energy allocated to immune defense (French et al., 2007). In this sense, our data support that the initial mite load was an important predictor of mite load increase at recapture. This suggests that mites may not increase in the lizards as the encounter rate between lizards and new mites increases, but instead as a function of the replication rate of mites on the lizards' body. In support of this hypothesis, our preliminary experiments of mites reared in captivity demonstrate that new cohorts of mites hatch within only 3-4 days after the mother mites complete a bloodmeal (Megía-Palma, pers. obs.). This can multiply mite loads on the lizards in only few days, supporting our hypothesis and making biological sense of the statistical results achieved in this study. The important aspect of these observations is that the results of our translocation experiment suggest that containing mite infestation is energetically demanding for $P$. algirus as their body condition decreases with the distance travelled, while mite load increases. Thus, energy invested in exploration seems to be traded by immune defense against mites. Our results would, thus, suggest that translocated lizards should be disinfested, as ectoparasite infestation may entail costs for hosts as they transmit hematic parasites and produce wounds in the skin surface, which can also be associated with mass loss (Smith et al., 2017; Megía-Palma et al., 2020). Other effects of severe ectoparasitic infestations in small to mid-sized lizards include alteration of the host's thermoregulatory behaviour (Megía-Palma et al., 2020), although the latter was not tested in our study

The lack of differences in the average number of days between capture and recapture both for treatments and areas is consistent with the idea that it was equally difficult to find and recapture control or translocated males, and that there were no differences between areas. It is possible that translocation effects are stronger due to reduced survival, but we have no data in this sense. However, given the symmetry in both the time invested in re-sampling effort between areas, and recapture ratio between treatment groups, a differential survivorship due to the soft translocation treatment is unlikely in the short time. Whereas we found a negative influence of the number of elapsed days between captures on distance covered, the former variable was positively related to the change in body condition. This is in line with the idea that translocated lizards increased their motor activity shortly after release, decreasing it afterwards, when they found a new territory to settle (Reinert and Rupert, 1999; Tuberville et al., 2005). Consequently, whereas mass loss is found in the short-term (Matthews, 2003), when translocated individuals find a new territory, they manage to increase their body mass in similar rates to control individuals (Reinert and Rupert, 1999; Brown et al., 2009). Although we are aware that we have not tested the survivorship of lizards after soft translocation, based on the results achieved, we recommend that individuals with below-average body condition should not be directly released in mitigationdriven translocations, especially in short-lived species, in which short-term body condition impoverishment may have greater impact. Our data are consistent with previous studies showing that male lizards, in average, loss weight during the reproductive season at low to moderate rates (Abell, 2000). Alternatively, we suggest that the implementation of soft releases (which allow the animals a period to acclimate to their new environment), at least for those individuals in poorer body condition, could 
minimize the costs of this management practice (e.g., Tuberville et al., 2005; Germano and Bishop, 2009). This pre-release management is designed so that animals in worse conditions can gain body mass.

In summary, although translocation protocols should be tailored to the target species and their habitats, being based on a thorough understanding of the species' biology and behaviour, including a long-term post-translocation monitoring (Tuberville et al., 2005; Germano et al., 2015), our study provides evidence that simulated mitigation-driven translocations result in increased exploratory behavior, decreased body condition, and increased parasite loads in a common lizard. If these issues are not taken into account, the success of mitigation-driven translocations may be compromised.

Acknowledgements. S. Reguera, C. Ponce, I. Pozo, M. Fernández, Z. Rohrer, C. Luque and P. Quiles helped with the field work. Z. Rohrer reviewed the language of the final version. Two anonymous reviewers improved a first draft. Handling protocols, as well as the general ethics of our research were approved by the ethic committee of University Complutense de Madrid (Ref. 5005), and by Comunidad de Madrid, Consejería de Medio Ambiente, Ordenación del Territorio y Sostenibilidad (Ref. 10/165944.9/18, PROEX 271/19) in accordance with current Spanish laws. RB enjoyed a postdoctoral grant from Comunidad de Madrid (2018T1/AMB10374), and RMP a postdoctoral contract by ICETA - Instituto de Ciências, Tecnologias e Agroambiente da Universidade do Porto and Fundação da Ciência e Tecnologia.

Supplementary material. Supplementary material is available online at: https://doi.org/10.6084/m9.figshare.14332955

\section{References}

Abell, A.J. (2000): Costs of reproduction in male lizards, Sceloporus virgatus. Oikos 88: 630-640.

Álvarez-Ruiz, L., Megía-Palma, R., Reguera, S., Ruiz, S., Zamora-Camacho, F.J., Figuerola, J., Moreno-Rueda, G. (2018): Opposed elevational variation in prevalence and intensity of endoparasites and their vectors in a lizard. Curr. Zool. 64: 197-204.

Barton, K. (2018): MuMIn: Multi-Model Inference. R package version 1.40.4. https://CRAN.R-project.org/ package $=$ MuMIn.
Belliure, J., Carrascal, L.M., Diaz, J.A. (1996): Covariation of thermal biology and foraging mode in two Mediterranean lacertid lizards. Ecology 77: 1163-1173.

Berger-Tal, O., Blumstein, D.T., Swaisgood, R.R. (2020): Conservation translocations: a review of common difficulties and promising directions. Anim. Conserv. 23: 121-131.

Brown, J.R., Bishop, C.A., Brooks, R.J. (2009): Effectiveness of short-distance translocation and its effects on western rattlesnakes. J. Wildlife Manage. 73: 419-425.

Buchanan, K. (2012): Guidelines for the treatment of animals in behavioural research and teaching. Anim. Behav. 83: 301-309.

Burnham, K.P., Anderson, D.R. (2004): Multimodel inference, understanding AIC and BIC in model selection. Sociol. Methods. Res. 33: 261-304.

Cote, J., Clobert, J., Meylan, S., Fitze, P.S. (2006): Experimental enhancement of corticosterone levels positively affects subsequent male survival. Horm. Behav. 49: 320327.

Dunlap, K.D., Mathies, T. (1993): Effects of nymphal ticks and their interaction with malaria on the physiology of male fence lizards. Copeia 1993: 1045-1048.

Eisen, L., Eisen, R.J., Lane, R.S. (2004): The roles of birds, lizards, and rodents as hosts for the western black-legged tick Ixodes pacificus. J. Vector Ecol. 29: 295-308.

Forman, R.T.T., Sperling, D., Bissonette, J.A., Clevenger, A.P., Cutshall, C.D., Dale, V.H., Fahrig, L., France, R., Goldman, C.R., Heanue, K., Jones, J.A., Swanson, F.J., Turrentine, T., Winter, T.C. (2003): Road Ecology: Science and Solutions. Island Press, Covelo and London.

French, S.S., McLemore, R., Vernon, B., Johnston, G.I., Moore, M.C. (2007): Corticosterone modulation of reproductive and immune systems trade-offs in female tree lizards: long-term corticosterone manipulations via injectable gelling material. J. Exp. Biol. 210: 2859-2865.

Germano, J.M., Bishop, P.J. (2009): Suitability of amphibians and reptiles for translocation. Conserv. Biol. 23: 7-15.

Germano, J.M., Field, K.J., Griffiths, R.A., Culow, S., Foster, J., Harding, G., Swaisgood, R.R. (2015): Mitigationdriven translocations: are we moving wildlife in the right direction? Front. Ecol. Environ. 13: 100-105.

Langkilde, T., Shine, R. (2006): How much stress do researchers inflict on their study animals? A case study using a scincid lizard, Eulamprus heatwolei. J. Exp. Biol. 209: 1035-1043.

Laurance, W.F., Clements, R.G., Sloan, S., O'Connell, C.S., Mueller, N.D., Goosem, M., Venter, O., Edwards, D.P., Phalan, B., Balmford, A., van Der Ree, R., Burgues Arrea, I. (2014): A global strategy for road building. Nature 513: 229-232.

Lewis, A.R., Saliva, J.E. (1987): Effects of sex and size on home range, dominance, and activity budgets in Ameiva exsul (Lacertilia: Teiidae). Herpetologica 43: 374-383.

Maindonald, J.H., Braun, W.J., Braun, M.W.J. (2015): Package 'DAAG'. Data Analysis and Graphics Data and Functions.

Martín, J., López, P. (1999): Nuptial coloration and mate guarding affect escape decisions of male lizards Psammodromus algirus. Ethology 105: 439-447. 
Matthews, K.R. (2003): Response of mountain yellowlegged frogs, Rana muscosa, to short distance translocation. J. Herpetol. 37: 621-626.

Maxwell, S.L., Fuller, R.A., Brooks, T.M., Watson, J.E.M. (2016): The ravages of guns, nets and bulldozers. Nature 536: 143-145.

Megía-Palma, R., Paranjpe, D., Blaimont, P., Cooper, R., Sinervo, B. (2020): To cool or not to cool? Intestinal coccidians disrupt the behavioural hypothermia of lizards in response to tick infestation. Ticks Tick-borne Dis. 11: 101275.

Meijer, J.R., Huijbregts, M.A.J., Schotten, K.C.G.J., Schipper, A.M. (2018): Global patterns of current and future road infrastructure. Environ. Res. Lett. 13: 604006.

Perry, G., Wallace, M.C., Perry, D., Curzer, H., Muhlberger, P. (2011): Toe clipping of amphibians and reptiles: science, ethics, and the law. J. Herpetol. 45: 547-555.

Reichenow, E. (1920): Los Hemococcidios de los lacértidos: estudio del desarrollo de karyolysus: observaciones previas y 1a parte: estudio y desarrollo de Karyolysus. Imprenta y Librería de Nicolás Moya.

Reinert, H.K., Rupert, R.R. (1999): Impact of translocation on behavior and survival of timber rattlesnake, Crotalus horridus. J. Herpetol. 33: 45-61.

Salvador, A., Veiga, J.P., Martín, J., López, P., Abelenda, M., Puerta, M. (1996): The cost of producing a sexual signal: testosterone increases the susceptibility of male lizards to ectoparasitic infestation. Behav. Ecol. 7: 145150.

Salvador, A. (2015): Lagartija Colilarga - Psammodromus algirus in Enciclopedia Virtual de los Vertebrados Españoles. Salvador A., Marco A., Eds. Museo Nacional de Ciencias Naturales, Madrid. Available from http://www.vertebradosibericos.org/ (Accessed 9th October 2020).

Smith, G.D., Neuman-Lee, L.A., Webb, A.C., Angilletta, M.J., DeNardo, D.F., French, S.S. (2017): Metabolic responses to different immune challenges and varying resource availability in the side-blotched lizard (Uta stansburiana). J. Comp. Physiol. B 187: 1173-1182.
Strijbosch, H., van Rooy, P.T.J., Voesenek, L.A.C.J. (1983): Homing behaviour of Lacerta agilis and Lacerta vivipara (Sauria, Lacertidae). Amphibia-Reptilia 4: 43-47.

Sullivan, B.K., Kwiatkowski, M.A., Chutt, G.W. (2004): Translocation of urban Gila Monsters: a problematic conservation tool. Biol. Conserv. 117: 235-242.

Taylor, G., Canessa, S., Clarke, R.H., Ingwersen, D., Armstrong, D.P., Seddon, P.J., Ewen, J.G. (2017): Is reintroduction biology an effective applied science? Trends Ecol. Evol. 32: 873-880.

Teixeira, C.P., De Azevedo, C.S., Mendl, M., Cipreste, C.F., Young, R.J. (2007): Revisiting translocation and reintroduction programmes: the importance of considering stress. Anim. Behav. 73: 1-13.

Tetzlaff, S.J., Sperry, J.H., DeGregorio, B.A. (2019): Effects of antipredator training, environmental enrichment, and soft release on wildlife translocations: a review and meta-analysis. Biol. Conserv. 236: 324-331.

Tuberville, T.D., Clark, E.E., Buhlmann, K.A., Gibbons, J.W. (2005): Translocation as a conservation tool: site fidelity and movement of repatriated gopher tortoises (Gopherus polyphemus). Anim. Conserv. 8: 349-358.

van der Ree, R., Smith, D.J., Grilo, C., Eds (2015): Handbook of Road Ecology. John Wiley and Sons, Chichester.

Wieczorek, M., Rektor, R., Najbar, B., Morelli, F. (2020): Tick parasitism is associated with home range area in the sand lizard, Lacerta agilis. Amphibia-Reptilia 1: 1-10.

Wu, Q., Richard, M., Rutschmann, A., Miles, D.B., Clobert, J. (2019): Environmental variation mediates the prevalence and co-occurrence of parasites in the common lizard, Zootoca vivipara. BMC Ecology 19: 44.

Submitted: May 1, 2020. Final revision received: December 2, 2020. Accepted: December 2, 2020.

Associate Editor: Sylvain Dubey. 